\title{
Exact solutions of the Newton-Schrödinger equation, infinite derivative gravity and Schwarzschild atoms
}

\begin{abstract}
Exact solutions to the stationary spherically symmetric Newton-Schrödinger equation are proposed in terms of integrals involving generalized Gaussians. The energy eigenvalues are also obtained in terms of these integrals which agree with the numerical results in the literature. A discussion of infinite derivative-gravity follows which allows generalizing the Newton-Schrödinger equation by replacing the ordinary Poisson equation with a modified non-local Poisson equation associated with infinite-derivative gravity. We proceed to replace the nonlinear Newton-Schrödinger equation for a non-linear quantum-like BohmPoisson equation involving Bohm's quantum potential, and where the fundamental quantity is no longer the wave-function $\Psi$ but the real-valued probability density $\rho$. Finally, we discuss how the latter equations reflect a nonlinear feeding loop mechanism between matter and geometry which allows us to envisage a "Schwarzschild atom" as a spherically symmetric probability cloud of matter which curves the geometry, and in turn, the geometry back-reacts on this matter cloud perturbing its initial distribution over the space, which in turn will affect the geometry, and so forth until static equilibrium is reached.
\end{abstract}

Keywords: quantum mechanics, Newton-Schrödinger equation, infinite derivative gravity, de Broglie-Bohm theory, Schwarzschild metric
Volume I Issue 4 - 2017

\author{
Carlos Castro Perelman \\ Center for Theoretical Studies of Physical Systems, Clark Atlanta \\ University, Georgia
}

\begin{abstract}
Correspondence: Carlos Castro Perelman, Center for Theoretical Studies of Physical Systems, Clark Atlanta University, Atlanta, GA 303I4, Georgia, Email perelmanc@hotmail.com
\end{abstract}

Received: October 07, 2017 | Published: November 10, 2017

\section{Introduction}

\section{The Newton-Schrödinger equation}

Various arguments have been put forward from time to time to support the view that quantum state reduction is a phenomenon that occurs objectively, because of some gravitational influence. ${ }^{1-6}$ According to a particular argument put forward by Penrose, ${ }^{1}$ a superposition of two quantum states, each of which would be stationary on its own, but for which there is a significant mass displacement between the two states, ought to be unstable and reduce to one state or the other within a certain characteristic average timescale $T_{G}$. This argument is motivated by a conflict between the basic principles of quantum mechanics and those of general relativity. It is accordingly proposed that $T_{G}$ can be calculated in situations for which velocities and gravitational potentials are small in relativistic units, so that a Newtonian approximation is appropriate, and $T_{G}$ is the reciprocal, in Planckian units, of the gravitational self-energy $E_{G}$ of the difference between the mass distributions of the two states.

There is of course a substantial literature on the problem of wave-function collapse and the related measurement problem. See, for example ${ }^{4-6}$ and references therein. For a different idea about gravitationally-induced wave-function collapse see. ${ }^{3}$ It has been pointed out by Penrose $\mathrm{R}$ et al..$^{1,2}$ that one can regard the basic stationary states, into which a superposition of such states is to decay into (on a timescale of order $\hbar / E_{G}$ ), as stationary solutions of the Schrödinger equation where there is an additional term provided by a certain gravitational potential. The appropriate gravitational potential is the one which arises from the mass density given by the expectation value of the mass distribution in the state determined by the wave-function. In the practical situations under consideration in ${ }^{1}$ (such as with the proposed class of experiments put forward there), it would be sufficient to consider Newtonian gravity. This leads us to consider what Penrose termed the Schrödinger-Newton equation. This equation has had a long history since the 1950 's. $^{7-17}$ It is the name given to the system coupling the Schrödinger equation to the Poisson equation. In the case of a single particle, this coupling is effected as follows: for the potential energy term in the Schrödinger equation take the gravitational potential energy determined by the Poisson equation from a matter density proportional to the probability density obtained from the wave-function. For a single particle of mass $m$ the system consists of the following pair of partial differential equations:

The Newton-Schrödinger equation is nonlinear and nonlocal modification of the Schrödinger equation given by

$$
i \hbar \frac{\partial \Psi(\vec{r}, t)}{\partial t}=-\frac{\hbar^{2}}{2 m} \nabla^{2} \Psi(\vec{r}, t)+V(\vec{r}, t) \Psi(\vec{r}, t)+m V_{G}(\vec{r}, t) \Psi(\vec{r}, t)
$$

Where $V(\vec{r}, t)$ is the external potential acting on the particle and $m V_{G}(\vec{r}, t)$ is the self- gravitational potential energy arising due a mass density obtained from the wave function of the particle itself. Given the Poisson equation sourced by a mass density $\rho=m|\Psi(\vec{r}, t)|^{2}$

$$
\nabla^{2} V_{G}(\vec{r}, t)=4 \pi G m|\Psi(\vec{r}, t)|^{2}
$$

It leads to a self-gravitational potential

$$
V_{G}(\vec{r}, t)=-G \int \frac{m|\Psi(\vec{r}, t)|^{2}}{\left|\vec{r}-\vec{r}^{\prime}\right|} d^{3} r^{\prime}
$$

Inserting equation (3) into equation (1) leads to the integro differential form of the nonlinear and 
nonlocal Newton-Schrödinger equation

$$
i \hbar \frac{\partial \Psi(\vec{r}, t)}{\partial t}=-\frac{\hbar^{2}}{2 m} \nabla^{2} \Psi(\vec{r}, t)+V(\vec{r}, t) \Psi(\vec{r}, t)-\left(G m^{2} \int \frac{m \mid \Psi(\vec{r}, t)^{2}}{\left|\vec{r}-\vec{r}^{\prime}\right|} d^{3} r^{\prime}\right) \Psi(\vec{r}, t)
$$

Relevant work pertaining pseudo differential and hyper differential operators, nonlinear partial differential equations and their applications can be found in. ${ }^{18-20}$

This equation is based on the assumption that the point-particle is smeared over space such that its mass is distributed according to its wave function. Namely, there is a mass cloud over space whose net mass $m=m \int|\Psi(\vec{r}, t)|^{2} d^{3} r^{\prime}$. coincides with the mass of the point-particle. The mass cloud is self-gravitating and experiences a gravitational potential energy given by equation (3).

More recently, the authors ${ }^{21}$ showed that nonlinear Schrödinger equations (NSEs) for individual particles do not follow from general relativity (GR) plus quantum field theory (QFT). Contrary to what is commonly assumed, the NSEs are not the weak-field, non-relativistic limit of the semi-classical Einstein equation. The wave-function in the NSEs makes sense only as that for a mean field describing a system of $\mathrm{N}$ particles as $N \rightarrow \infty$, not that of a single or finite many particles. They concluded that the origins and consequences of NSEs are very different, and should be clearly demarcated from those of the semiclassical Einstein equation, the only legitimate representative of semi classical gravity, based on GR+QFT. Bearing this in mind, we proceed to find solutions to the NSE's.

\section{Solutions to the Newton-Schrödinger equation}

Let us set the external potential to zero and look for stationary solutions $\Psi(\vec{r}, \mathrm{t})=\mathrm{e}^{-\mathrm{iEt} / \hbar} \Phi(\vec{r})$ such that the gravitational potential becomes time independent. Ö $(\vec{r})$ obeys the equation

$$
E \ddot{O}(\vec{r})=-\frac{\hbar^{2}}{2 m} \nabla^{2} \ddot{O}(\vec{r})-\left(G m^{2} \int \frac{|\ddot{\mathrm{O}}(\vec{r})|^{2}}{\left|\vec{r}-\vec{r}^{\prime}\right|} d^{3} r^{\prime}\right) \ddot{\mathrm{O}}(\vec{r})
$$

The authors ${ }^{22-26}$ found numerical spherically-symmetric solutions to equation (5). Variational forms of the stationary NewtonSchrödinger equation to find a lower bound for the ground state energy have been studied by several authors, see references in, ${ }^{27}$ and compared to numerical values in the literature.

${ }^{1}$ This may be in conflict with Born's rule of interpreting $|\Psi(\vec{r}, t)|^{2}$ as the probability density of finding a particle at the point $\vec{r}$ if one has abandoned the notion of point-particles. At the moment we shall not be concerned with this.

If one replaces a delta function point-mass source distribution $m \delta^{3}(\vec{r})=m \delta(r) / 4 \pi r^{2}$ for a normalized Gaussian mass distribution of width

$$
\rho(r)=\frac{m}{\pi^{3 / 2}} \frac{e^{-r^{2} / \sigma^{2}}}{\sigma^{3}} \Rightarrow m=\int_{0}^{\infty} \rho(r) 4 \pi r^{2} d r
$$

The solution to Poisson's equation

$$
\nabla^{2} V_{G}(r)=4 \pi G \rho(r)
$$

Is given in terms of the error function $\operatorname{Erf}(r)^{2}$ as follows

$$
V_{G}(r)=-\frac{G m}{r} \operatorname{Erf}\left(\frac{r}{\sigma}\right)=-\frac{G m}{r} \frac{1}{\sqrt{\pi}} \int_{-r / \sigma}^{r / \sigma} e^{-t^{2}} d t
$$

In the asymptotic regime $r \rightarrow \infty, \operatorname{Erf}(\infty) \rightarrow 1$, the potential recovers the Newtonian form $-\frac{G m^{2}}{r}$. Because the error function $\operatorname{Erf}(r)$ admits a series expansion around $r=0$ as

$$
\operatorname{Erf}(r) \simeq \frac{2 r}{\sqrt{\pi}}-\frac{r^{3}}{\sqrt{\pi}}+\frac{11 r^{5}}{20 \sqrt{\pi}}-\frac{241 r^{7}}{840 \sqrt{\pi}}+\ldots
$$

The potential $V_{G}(r)$ is no longer singular at the origin $r=0$, but it behaves as

$$
V_{G}(r) \simeq-\frac{2 G m}{\sqrt{\pi} \sigma}\left[1-\frac{r^{2}}{2 \sigma^{2}}+\frac{11 r^{4}}{40 \sigma^{4}}+\ldots\right]
$$

The normalized Gaussian wave function

$$
\Phi(\vec{r}) \equiv \frac{1}{\pi^{3 / 4}} \frac{e^{-r^{2} / 2 \sigma^{2}}}{\sigma^{3 / 2}}
$$

Satisfies

$$
\int_{0}^{\infty}|\Phi(\vec{r})|^{2} 4 \pi r^{2} d r=1
$$

Let us minimize the energy functional

$$
E=\frac{\hbar^{2}}{2 m} \int_{0}^{\infty}\left(\Phi(\vec{r}) \nabla^{2} \Phi(\vec{r})\right) 4 \pi r^{2} d r+\int_{0}^{\infty} m V_{G}[\Phi(\vec{r})]|\Phi(\vec{r})|^{2} 4 \pi r^{2} d r
$$

Using this Gaussian as a trial function, and which generates the regular potential at the origin $V_{G}[\Phi(\vec{r})]=\frac{G m}{r} \operatorname{Erf}\left(\frac{r}{\sigma}\right)$ given by equation (8) after solving Poisson's equation (7). ${ }^{2}$ The integrals to be evaluated in equation (13) are of the form

$$
\int_{0}^{\infty}\left(x^{4}-3 x^{2}\right) \mathrm{e}^{-x^{2}} d x=-\frac{3}{8} \sqrt{\pi} \operatorname{Erf}(x)-\frac{1}{4} \mathrm{e}^{-x^{2}} x\left(2 x^{2}-3\right)
$$

${ }^{2}$ Which is also related to the incomplete gamma function $\gamma\left(\frac{1}{2} ; r\right)=\sqrt{\pi} \operatorname{Erf}(r)$

$$
\int_{0}^{\infty} x \operatorname{Erf}(x) \mathrm{e}^{-x^{2}} d x=\frac{1}{4}\left(\sqrt{2} \operatorname{Erf}(\sqrt{2} x)-2 \mathrm{e}^{-x^{2}} \operatorname{Erf}(x)\right)
$$

After performing the definite integrals we find that

$$
\begin{gathered}
E(\sigma)=\frac{3 \sqrt{\pi}}{4} \frac{\hbar^{2}}{m \sigma^{2}}-\sqrt{\frac{2}{\pi}} \frac{G m^{2}}{\sigma} \\
\sigma_{\text {min }}=\frac{3 \pi}{2 \sqrt{2}} \frac{\hbar^{2}}{G m^{3}}
\end{gathered}
$$

And inserting this value of $\sigma$ into $E(\sigma)$ gives

$$
E_{\text {min }}=\frac{2}{3 \pi^{3 / 2}} \frac{G^{2} m^{5}}{\hbar^{2}}=-0.119 \frac{G^{2} m^{5}}{\hbar^{2}}
$$

Which is a satisfactory value since it is above the lower energy bound

$$
E_{\text {bound }}=\frac{32}{9 \pi^{2}} \frac{G^{2} m^{5}}{\hbar^{2}}=-0.360 \frac{G^{2} m^{5}}{\hbar^{2}}
$$

Derived by. ${ }^{27}$ and it is also close to the value $-0.163\left(G^{2} \mathrm{~m}^{5} / \hbar^{2}\right)$ obtained numerically by. ${ }^{22-26}$ We can also verify that the value of $\sigma_{\text {min }}$ given by equation (17) is consistent with the virial theorem $|U|=2 T$, stating that the absolute value of the potential energy 
is twice the kinetic energy. It is also worth mentioning that a trial exponential $\psi(r)=\left(k^{3} / \pi\right)^{1 / 2} e^{-k r}$ leads to an upper energy bound for the ground state of ${ }^{27}$

$$
E_{0} \leq-\frac{75}{512} \frac{G^{2} m^{5}}{\hbar^{2}}=-0.146 \frac{G^{2} m^{5}}{\hbar^{2}}
$$

Which is close to the value found numerically by. ${ }^{22-26}$ However the series expansion of $e^{-k r}$ involves even and odd powers of $r$ which is not the case of the numerical solutions provided by. ${ }^{22-26}$ The spherically-symmetric solutions of the stationary SchrödingerNewton equations have been solved numerically by many authors. ${ }^{22-26}$ They have demonstrated numerically the existence of a discrete set of "bound-state" solutions which are everywhere finite and smooth (and which are associated with finite energy eigenvalues), but which separate ever decreasing intervals of partial solutions which diverge alternately to plus or minus infinity.

The value found for the ground state energy turned out to be

$$
E_{0}=-0.163 \frac{G^{2} m^{5}}{\hbar^{2}}=-0.163\left(\frac{m}{M_{\text {planck }}}\right)^{5} M_{\text {planck }} c^{2}
$$

These numerical values for the energy are very small for masses much smaller than the Planck mass.

The width (spread) $\sigma$ of the Gaussian wave-function is measured in units of $\left(\hbar^{2} / G^{2} m^{5}\right)$, which translated into Planckian units is $\left(L_{P} / L_{S}\right)^{3}\left(2 L_{P}\right)$, where $L_{P}$ is the Planck length $\left(2 \hbar G / c^{3}\right)^{1 / 2}$ , and $L_{S}=2 \mathrm{Gm} / \mathrm{c}^{2}$ is the $S$ chwarzschild radius. It was argued by the authors ${ }^{22}$ that for a nucleon mass $m$ the value of $\sigma$ is vast and is of order of $10^{24} \mathrm{~cm}$. The corresponding time $\Delta t=\hbar / \Delta E$ for the largest (in magnitude) energy is about of $10^{53}$ seconds for the mass of the nucleon, and is of the order of 1 second for $10^{11}$ nucleon masses. This is perfectly satisfactory for the state vector reduction of, ${ }^{1}$ because it tells us that the state of a single nucleon will not self-reduce on a timescale of relevance to any actual particle, in agreement with observation. For large collections of particles, on the other hand, the reduction time can become important.

The coupled system of differential equations $(1,2)$ can be recast after integrating twice, and using dimensionless variables $S, V$, as ${ }^{22}$

$$
\begin{aligned}
& S(r)=\mathrm{S}_{0}-\frac{1}{l^{2}} \int_{0}^{r} x\left(1-\frac{x}{r}\right) S(x) V(x) d x \\
& V(r)=V_{0}-\frac{1}{l^{2}} \int_{0}^{r} x\left(1-\frac{x}{r}\right) S^{2}(x) d x
\end{aligned}
$$

The value of $V_{0}$ must be $V_{0}>0$ to ensure convergence of $S(r)$ and $V(r)$ at $r \rightarrow \infty$. Scaling arguments allow one to choose $V_{0}=1$. The numerical solutions to equations $(22,23)$ found by, ${ }^{22}$ when $V_{0}=1$ , are

$$
\begin{gathered}
S(r)=\mathrm{S}_{0}\left(1-\frac{1}{6} \frac{r^{2}}{l^{2}}+\frac{\left(S_{0}^{2}+1\right)}{120} \frac{r^{4}}{l^{4}}+\ldots\right) \\
V(r)=\left(1-\frac{S_{0}^{2}}{6} \frac{r^{2}}{l^{2}}+\frac{S_{0}^{2}}{60} \frac{r^{4}}{l^{4}}+\ldots\right)
\end{gathered}
$$

The wave-functions are $\Psi(r)=\frac{\alpha}{l^{2}} S(r)$, and where $\alpha, \beta$ are

$$
\alpha \equiv\left[\frac{\hbar^{2}}{8 \pi G m^{2}}\right]^{1 / 2}, \beta \equiv \frac{\hbar^{2}}{2 m}
$$

And $l$ is a physical length parameter (the gravitational analog of the Bohr's radius) given by $l=\hbar^{2} / G m^{3}$, such that $\beta / l^{2}=\frac{1}{2}\left(G^{2} m^{5} / \hbar^{2}\right)$,

The energy eigenvalues are $E=U(r)+\frac{\beta}{l^{2}} V(r)$. Because $U(r=0)$ is of the form $\infty \times 0$ which is undefined, it is more convenient to evaluate the energy at $r=\infty$ where $U(\infty)$ vanishes, as follows $22-26,28$

$$
\mathrm{E}=U(r=\infty)+\frac{\beta}{l^{2}} V(r=\infty)=\frac{\beta}{l^{2}}\left(V_{0}-\frac{1}{l^{2}} \int_{0}^{\infty} x S^{2}(x) d x\right), V_{0}=1
$$

The values of $\mathrm{S}_{0}$ are fine-tuned such that the ground state wavefunction has no zeros, it is bounded at the origin and vanishes at infinity. By demanding that $\Psi$ and $U$ are finite and smooth everywhere, the authors showed that the $n$-th eigen function has $n$ zeros and the wavefunctions are normalizable. The corresponding energy eigenvalues are negative, converging monotonically to zero as $n$ increases. Each bound-state is unstable in the sense that infinite precision is required in the initial value of $S(r=0)=\mathrm{S}$ to ensure that the solutions do not diverge to infinity as $r$ increases. For the ground state, the numerical value of $\mathrm{S}_{0}$ fell in the range given by $1.088<\mathrm{S}_{0}<1.090$ and it led to the ground state energy $E_{0}=-0.163 \frac{G^{2} m^{5}}{\hbar^{2}}$.

For further details of how to obtain other energy eigen values and eigen functions by choosing different values for $\mathrm{S}$ we refer to. ${ }^{23-36}$ The study of the Newton-Schrödinger equations in $D$ dimensions other than $D=3$ can be found in. ${ }^{28}$

A careful inspection of the work by ${ }^{22-26,28}$ inspired us to find the remarkable numerical coincidence provided by the definite integral

$$
-\int_{0}^{\infty} e^{-\left(y^{2}+y^{4}\right)} y^{2} d y=-0.160 \simeq-0.163=\frac{1}{2}\left(1-\frac{1}{l^{2}} \int_{0}^{\infty} x S^{2}(x) d x\right)
$$

(We $\operatorname{set} V_{o}=1$ ). The normalization of the wave function $\Psi=\frac{\alpha}{l^{2}} S(r)$

$$
\frac{\alpha^{2}}{l^{4}} \int_{0}^{\infty} 4 \pi r^{2} S^{2} d r=1
$$

Allows to recast the right-hand side of (28) as

$$
\frac{\alpha^{2}}{2 l^{4}} \int_{0}^{\infty} 4 \pi r^{2} S^{2} d r-\frac{1}{2 l^{2}} \int_{0}^{\infty} x S^{2}(x) d x
$$

After relabeling the variable $r$ for $x$ in the above expression, equation (28) becomes

$$
-0.160=-\int_{0}^{\infty} e^{-\left(y^{2}+y^{4}\right)} y^{2} d y \simeq \frac{1}{2} \int_{0}^{\infty}\left(\frac{4 \pi \alpha^{2}}{l} y-1\right) y S^{2}(y) d y=-0.163
$$

Which allows to identify the variables $y \equiv \frac{x}{l}=\frac{r}{l}$, and basically equate the dimensionless integrals. However this does not mean that the integrands are the same. The integral in the right-hand side of equation (30) is evaluated after inserting the value of $4 \pi \alpha^{2} / l=\frac{1}{2}$ The expression for $S^{2}(r)$ corresponding to the ground state is 
derived from equation (24) after inserting a value of $S \simeq 1.088$ (lying in the interval[1.088,1.090]). The other "bound" states require different values of $S$ yielding different expressions for $S_{n}(r)\left(S_{n}(r=0)=S_{o, n}\right)$, and which have $n$ nodes (zeros). Next we shall present the novel and most salient results of this section. To our knowledge these findings below are new. The following remarkable numerical coincidences for the values of the remaining (negative) energy eigenvalues $E_{1}, E_{2}, E_{3}, \ldots$. have also been found. To obtain the values for the first excited state we replace the integral in the lefthand side of (28) for:

$$
n=1:-\frac{1}{2^{2}} \int_{0}^{\infty} e^{-\left(y^{2}+y^{4}+y^{6}\right)} y^{2} d y=-0.0302 \simeq-0.0308
$$

To get the second one

$$
n=2:-\frac{1}{3^{2}} \int_{0}^{\infty} e^{-\left(y^{2}+y^{4}+y^{6}+y^{8}\right)} y^{2} d y=-0.0120 \simeq-0.0125
$$

The third one

$$
n=3:-\frac{1}{4} \int_{0}^{\infty} e^{-\left(y^{2}+y^{4}+y^{6}+y^{8}+y^{10}\right)} y^{2} d y=-0.00641 \simeq-0.00675
$$

The fourth one

$$
n=4:-\frac{1}{5^{2}} \int_{0}^{\infty} e^{-\left(y^{2}+y^{4}+y^{6}+y^{8}+y^{10}+y^{12}\right)} y^{2} d y=-0.00399 \simeq-0.00421
$$

And so forth.... The pre-factors in front of the integrals for the $n$-th energy eigenvalue are $(n+1)^{-2}$, which are "reminiscent" of the Balmer series for the Hydrogen atom, and the arguments of the negative exponentials are $y^{2}+y^{4}+\cdots+y^{2(n+2)}$. The authors ${ }^{22}$ plotted the ten energy eigenvalues as functions of $n$ and found that the slope of $\log (n)$ versus $\log (E(n))$ was not $-2 . .^{22}$ However, the slope is asymptotically close to $-2 .{ }^{35}$ which would be the exact value for the Hydrogen atom as previously note by Bernstein et al. ${ }^{23-26}$

Using these expressions in equation (28), and equations (31-34), we find that energy values are remarkably close to the numerical results obtained in, ${ }^{35}$ and given by the numbers in the right-hand side of equations $(28,31-34)$. We don't believe this is a numerical coincidence and could point to the fact that integrability may underlie the solutions of the stationary spherically symmetric NewtonSchrödinger equations. It was emphasized by ${ }^{22-26,28}$ that one would need to know the values of $S_{n}(r=0)=S_{o, n}$ with infinite precision in order to ensure that $S(r)$ does not shoot off to $\pm \infty$ at a finite value of $r$. All the numerical results appearing in the right-hand side of equations $(28,31-34)$ are obtained by narrowing in the values of $S$ within certain domains. For this reason we find the analytical results in the left-hand side of equations (28,31-34) very appealing.

To conclude this section we shall recur to the virial theorem $<U>=-2<T>\Rightarrow E=<T+U>=-<T>$ to write the most general expression for the energy after integration by parts, in the form

$$
\begin{aligned}
& E_{n}=-<T>_{n}=\frac{\hbar^{2}}{2 m} \int_{-\infty}^{\infty}\left(\Psi_{n}(\vec{r})\right)^{*} \nabla^{2} \Psi_{n}(\vec{r}) d^{3} r=-\frac{\hbar^{2}}{2 m} \int_{-\infty}^{\infty}\left|\left(\nabla \Psi_{n}(\vec{r})\right)\right|^{2} d^{3} r \simeq \\
& -\frac{1}{(n+1)^{2}} \frac{G^{2} m^{5}}{\hbar^{2}} \int_{0}^{\infty} e^{-\left(y^{2}+y^{4}+y^{6}+\cdots+y^{2(n+2)}\right)} y^{2} d y
\end{aligned}
$$

The wave-functions must be normalized and vanish at $\pm \infty$. One must emphasize that one is comparing (roughly equating) the values of the integrals in (35) and not the integrands.

It is clear that one cannot equate the integrands in equation (35). By a simple inspection one can see that there are zeros (besides $r=0$
) in the left hand side, while in the right hand side one has zeros only at $r=0$. If one were to equate the integrands one would arrive at another contradiction. In the spherically symmetric case, for realvalued wave-functions, and after setting $y=r / l$, it gives

$$
\begin{aligned}
& \frac{\hbar^{2} l}{2 m}\left(\partial_{y} \Psi_{n}(y)\right)^{2} 4 \pi y^{2} \simeq \frac{1}{(n+1)^{2}} \frac{G^{2} m^{5}}{\hbar^{2}} e^{-\left(y^{2}+y^{4}+y^{6}+\cdots+y^{2(n+2)}\right)} y^{2} \Rightarrow \\
& \left(\partial_{y} \Psi_{n}(y)\right)^{2} \simeq \frac{1}{(n+1)^{2}} \frac{1}{2 \pi l^{3}} e^{-\left(y^{2}+y^{4}+y^{6}+\cdots+y^{2(n+2)}\right)}
\end{aligned}
$$

Given $\Psi$ an even, or an odd function, for $n=$ even, odd, respectively, the left hand side is always even due to the squaring of the derivatives, as it should, since the right hand side of (36) is an even functions. ${ }^{3}$ For this reason, if one is going to take the square root of equation (36) we must choose $n=$ odd $\Rightarrow \partial \Psi=$ even. Hence

$$
\partial_{y} \Psi_{n}(y) \simeq \frac{1}{(n+1)} \frac{1}{\sqrt{2 \pi l^{3}}} e^{\frac{-1}{2}\left(y^{2}+y^{4}+y^{6}+\cdots+y^{2(n+2)}\right)}, n=o d d
$$

Integrating (37) between 0 and $y$ we arrive finally

$$
\Psi_{n}(y)-\Psi_{n}(0) \simeq \frac{1}{(n+1)} \frac{1}{\sqrt{2 \pi l^{3}}} \int_{0}^{y} e^{\frac{-1}{2}\left(y^{2}+y^{4}+y^{6}+\cdots+y^{2(n+2)}\right)} d y, n=\text { odd }(38)
$$

We still need to check that vanishes at, and include normalization constant to enforce . $\int|\Psi|^{2} d^{3} r=1$. When $\mathrm{n}=$ odd, $\Psi(0)=0$ because anti symmetric functions must vanish at the origin. 3This assumes that one can extend to extend $r$ to negative values $r<0$ , which is not unreasonable because $r= \pm \sqrt{x^{2}+y^{2}+z^{2}}$. Black-hole solutions can be extended to $r<0$. The Schwarzschild metric solution is invariant under $r \rightarrow-r, m \rightarrow-m$.

Here is where the contradiction arises. The integral in the righthand side is not zero when $y=\infty$. It is given by a finite number. This would force $\Psi(\infty) \neq 0$ since $\Psi(0)=0$, for $n=$ odd. Since the wave-functions ${ }^{n}$ do not vanish at $\infty^{n}$ these solutions are unphysical. For this reason one cannot equate the integrands in equation (35) only the integrals which can be equated.

To conclude this section, based on the numerical results by $\mathrm{y}^{22-26,28}$ we have arrived at the integrals.

$$
\int_{0}^{y}\left(\partial_{y} \Psi_{n}(y)\right)^{2} y^{2} d y \simeq \frac{1}{(n+1)^{2}} \frac{1}{2 \pi l^{3}} \int_{0}^{y} e^{-\left(y^{2}+y^{4}+y^{6}+\cdots+y^{2(n+2)}\right)} y^{2} d y, n=0,1,2, \ldots
$$

Which furnish implicitly the stationary spherically symmetric wave-function solutions to the Newton-Schrödinger equation in terms of integrals involving generalized Gaussians. The energy eigen values are provided by the left hand side of equations (28,31-34). Equations (39) must be supplemented by the normalization condition of the wave-function. The time-dependent evolution of the SchrödingerNewton equations has been studied by many authors, in particular. ${ }^{36-39}$ The experimental tests of the validity of the nonlinear NewtonSchrödinger equation pose a technologically formidable challenge due to the weakness of gravity and the difficulty of controlling quantum coherence. ${ }^{39}$

\section{Infinite derivative gravity, modified Newton-Schrödinger equation and Schwarzschild atoms}

Since Quantum Mechanics is notoriously non-local, it is not farfetched that a theory of Quantum Gravity may require modifying Einstein's theory of gravity (and other local theories of gravitation) to include a modified gravitational theory involving infinite derivatives, 
which by construction, is non-local. It turned out that the infinite derivative gravity (IDG) can resolve the problem of massive ghosts as well as it may avoid the singularity of the Newtonian potential at the origin, when one chooses the exponential of an entire function. This model is also named super-renormalizable quantum gravity. ${ }^{40-42}$ However, one does not understand fully how this non-local gravity could provide a regular potential. It was argued that the cancellation of the singularity at the origin is an effect of an infinite amount of hidden ghost-like complex poles. ${ }^{42,43}$

A regular potential at the origin of the form (8) has been studied by several authors ${ }^{44}$ in connection to infinite derivative gravity ${ }^{42,43}$ which is ghost-free and renormalizable when one chooses the exponential of an entire function in the construction of the infinitederivative gravitational (IDG) action $S_{I D G}{ }^{42,43}$ For this IDG case, the corresponding Newtonian potential generated from the delta function is non-singular at the origin. However, the authors ${ }^{44}$ explicitly showed that the source generating this non-singular potential is given not by the delta-function due to the point-like source of mass, but by the Gaussian mass distribution. This explains clearly why the IDG with the exponential of an entire function yields the finite potential at the origin. The infinite-derivative (non-local) modified Poisson equation associated to a point-mass particle at $\vec{r}=0$ is given by ${ }^{42-44}$

$$
\left(e^{-\frac{\sigma^{2}}{4} \nabla^{2}} \nabla^{2}\right) V=4 \pi G m \delta^{3}(\vec{r})=G m \frac{\delta(r)}{r^{2}}
$$

$\sigma$ Is a length scale which can be taken to be equal to the Planckscale. Such equation (40) leads to identical solutions for the potential $V$ as the solutions for the potential in the ordinary Poisson equation sourced by a Gaussian mass distribution, ${ }^{44}$ and which is a smeared version of the delta function mass distribution. This can be shown by taking the $3 D$ Fourier transform of equation (40)

$$
e^{\frac{\sigma^{2}}{4} k^{2}} k^{2} \tilde{V}(k)=G m \Rightarrow \tilde{V}(k)=\frac{G m e^{-\frac{\sigma^{2}}{4} k^{2}}}{k^{2}}
$$

And then performing the inverse $3 D$ Fourier transform it yields $V_{G}(r)=-\frac{G m}{r} \operatorname{Erf}\left(\frac{r}{\sigma}\right)$, see $^{44}$ for further details. One can generalize the Newton-Schrödinger coupled system of equations $(1,2)$ by replacing the ordinary Poisson equation with the modified non-local Poisson equation associated with infinite-derivative gravity (IDG)

$$
\left(e^{-\frac{\sigma^{2}}{4} \nabla^{2}} \nabla^{2}\right) V_{I D G}(\vec{r})=4 \pi G \rho=4 \pi G m|\Phi(\vec{r})|^{2}
$$

In this case

$$
V_{I D G}(\vec{r}) \not G m \int \frac{|\Phi(\vec{r})|^{2}}{|\vec{r}-\vec{r}|} d^{3} r^{\prime}
$$

Therefore to find (numerical) solutions of the highly nonlinear and nonlocal modified Newton-Schrödinger equation

$$
E \Phi(\vec{r})=-\frac{\hbar^{2}}{2 m} \nabla^{2} \Phi(\vec{r})+m V_{I D G}[\Phi(\vec{r})] \Phi(\vec{r})
$$

Becomes more problematic. However, solutions to the infinite-derivative modified Poisson equation are not that difficult to find. For example, in the case when $\rho(r)$ is given by a Gaussian profile, after performing the Fourier transform procedure it gives $V_{I D G}=-\frac{G m^{2}}{r} \operatorname{Erf}\left(\frac{r}{\sqrt{2} \sigma}\right)$, which is almost identical to $-\frac{G m^{2}}{r} \operatorname{Erf}\left(\frac{r}{\sigma}\right)$, the main difference being that $\operatorname{Erf}\left(\frac{r}{\sigma}\right) \neq \operatorname{Erf}\left(\frac{r}{\sqrt{2} \sigma}\right)$

Bohm's quantum potential $V_{Q}=-\frac{\hbar}{2 m}(\nabla \sqrt{\rho} / \sqrt{\rho})$ has a geometrical description as the Weyl scalar curvature produced by an ensemble density of paths associated with one, and only one particle. ${ }^{45,46}$ This geometrization process of quantum mechanics allowed to derive the Schrödinger, Klein-Gordon ${ }^{45,46}$ and Dirac equations. ${ }^{47,48}$ Most recently, a related geometrization of quantum mechanics was proposed $^{49}$ that describe the time evolution of particles as geodesic lines in a curved space, whose curvature is induced by the quantum potential. This formulation allows therefore the incorporation of all quantum effects into the geometry of space-time, as it is the case for gravitation in the general relativity. Based on these results we propose the following nonlinear quantum-like Bohm-Poisson equation for static solutions $\rho=\rho(\vec{r})$.

$$
\nabla^{2} V_{Q}=4 \pi G m \rho \Rightarrow-\frac{\hbar^{2}}{2 m} \nabla^{2}\left(\frac{\nabla^{2} \sqrt{\rho}}{\sqrt{\rho}}\right)=4 \pi G m \rho
$$

Such that one could replace the nonlinear Newton-Schrödinger equation for the above non-linear quantum-like Bohm-Poisson equation (45) where the fundamental quantity is no longer the wave-function $\Psi$ (complex-valued in general) but the real-valued probability density $\rho=\Psi^{*} \Psi$.

It has been proposed by ${ }^{29-31}$ to give up the description of physical states in terms of ensembles of state vectors with various probabilities, relying instead solely on the density matrix as the description of reality. The time evolution of $\rho$ is governed by the Lindblad equation. ${ }^{4}$ The authors ${ }^{31}$ also investigated a number of unexplored features of quantum theory, including an interesting geometrical structure- which they called subsystem space-that they believed merits further study.

The infinite-derivative-gravity generalization of equation (45) is

$$
-\frac{\hbar^{2}}{2 m}\left(e^{-\sigma^{2} 4 \nabla^{2}} \nabla^{2}\right)\left(\frac{\nabla^{2} \sqrt{\rho}}{\sqrt{\rho}}\right)=4 \pi G m \rho
$$

The above equation is nonlinear and nonlocal. If one wishes to introduce a temporal evolution to $\rho$ via a Linblad-like equation, for instance, this would lead to an over determined system of differential equations for $\rho(\vec{r}, t)$. This problem might be another manifestation of the problem of time in Quantum Gravity. Naively replacing $\nabla^{2}$ in equations (45-46) for the D'Alambertian operator $\nabla \nabla^{\mu}, \mu=0,1,2,3$ has the caveat that in QFT $\rho\left(x^{\mu}\right)$ no longer has the interpretation of a probability density (it is now related to the particle number current). For the time being we shall just focus on static solutions $\rho(\vec{r})$. Integrating (45) gives the integral-differential equation for $\rho(\vec{r})$

$$
-\frac{\hbar^{2}}{2 m}\left(\frac{\nabla^{2} \sqrt{\rho}}{\sqrt{\rho}}\right)=-G m \int \frac{\rho\left(\vec{r}^{\prime}\right)}{\left|\vec{r}-\vec{r}^{\prime}\right|} d^{3} r^{\prime}
$$

${ }^{4}$ To be more precise it is the Gorini-Kossakowski-SudarshanLindblad equation

The right hand side of (47) can be written as

$$
-\frac{G m M_{e f f}(\vec{r})}{r}, M_{e f f}(\vec{r})=r \int \frac{\rho\left(\vec{r}^{\prime}\right)}{\left|\vec{r}-\vec{r}^{\prime}\right|} d^{3} r^{\prime}
$$


Where extreme caution must be taken because $M_{\text {eff }}(\vec{r})$ is not the same as the enclosed mass inside a spherical region of radius $r$ encircling the origin. It is convenient to write (47) in this form (48) because it reminds us of a "Schwarzschild atom" analog in the spherically symmetric case, where the $g$ metric component for signature $(+,-,-,-)$, and $c=1$, is

$$
g_{00}(r)=\left(1-\frac{2 G M_{e f f}(r)}{r}\right), g_{r r}=-\frac{1}{g_{00}(r)}, M_{e f f}(\vec{r})=M_{e f f}(r), c=1
$$

$\mathrm{See}^{33,34}$ for many references related to the solutions (49). In the weak field limit, for slowing moving masses, one has $g_{00} \sim \eta_{00}+h=1+2 V \quad(c=1)$, and from equations (47-49) one arrives at the relation between the temporal components of $h_{\mu v}$ and the matter/probability distribution

$$
h_{00}(r)=-\hbar^{2} m^{2}\left(\frac{\nabla^{2} \sqrt{\rho}(r)}{\sqrt{\rho}(r)}\right)=-\frac{2 G M_{e f f}(r)}{r}, c=1
$$

The equations (45-50) reflect the back-reaction that the geometry has no matter, and in turn, the effect that this matter has on the geometry. Such equations (45-50) reflect a nonlinear feeding loop mechanism between matter and geometry. The probability distribution $\rho$ cannot be arbitrary due to the restrictions imposed by equations $(45,46)$. One may envisage the "Schwarzschild atom" as a spherically symmetric probability cloud of matter which curves the geometry, and in turn, the geometry back-reacts on this matter cloud perturbing its initial distribution over the space, which in turn will affect the geometry, and so forth until static equilibrium is reached. The author ${ }^{32}$ described the gravitational degrees of freedom of the Schwarzschild black hole by one free variable and introduced a "Schrödinger equation" for the Schwarzschild black hole corresponding to this model. The mass spectrum of the black hole was obtained as such as it can be observed by an observer very far away and at rest relative to the black hole. Such "Schrödinger equation" implied that there is no singularity inside the Schwarzschild black hole, and that the black hole has a certain ground state in which its mass is non-zero. A modern treatment of this approach can be found in. ${ }^{33,34}$ We believe the picture of this "Schwarzschild atom" model will cast some light into Quantum Gravity.

\section{Conclusion}

Exact solutions to the stationary spherically symmetric NewtonSchrödinger equation were proposed in terms of integrals involving generalized Gaussians. The energy eigenvalues were also obtained in terms of these integrals which agree with the numerical results in the literature. A discussion of infinite-derivative-gravity followed which allows generalizing the Newton-Schrödinger equation by replacing the ordinary Poisson equation with a modified non-local Poisson equation associated with infinite-derivative gravity. Finally we proceeded to replace the nonlinear Newton-Schrödinger equation for a non-linear quantum-like Bohm-Poisson equation involving Bohm's quantum potential, and where the fundamental quantity is no longer the wave-function $\Psi$ but the real-valued probability density $\rho$.

Recently, solutions to the nonlinear Bohm-Poisson equation were found ${ }^{50}$ with cosmological applications. We envisioned the present Universe's matter density distribution as being proportional to the probability density, in the same vain that one can view an electron orbiting the Hydrogen nucleus as an "electron probability cloud" surrounding the nucleus, and whose mass density distribution is $\rho=m \Psi^{*} \Psi$, where $\Psi(\vec{r})$ the stationary wave-function solutions to the Schrödinger equation are. In doing so we were able to obtain the observed vacuum energy density after introducing an ultraviolet (very close to the Planck scale) and an infrared (Hubble radius) scale, and explained the origins of its repulsive gravitational nature. By inspection one can see that if $\rho(r)$ is a solution to the Bohm-Poisson equation (45) with $G>0$, and then $-\rho$ is a solution with $-G<0$. A negative gravitational constant is tantamount to repulsive gravity. For this reason, we believe that the Bohm-Poisson equation (45), and its relativistic generalization, deserves further investigations.

\section{Acknowledgments}

We thank M. Bowers for her assistance and to the referee for providing important references.

\section{Conflicts of Interest}

Author Declare there is no conflict of interest.

\section{References}

1. P $\backslash$ enrose R. On gravity's role in quantum state reduction. General Relativity and Gravitation. 1996;28(5):581-600.

2. Diosi L. Gravitation and quantum mechanical localization of macro objects. Physics Letters A. 1984;105(4-5):199-202.

3. Adler SL. Comments on proposed gravitational modifications of Schrödinger dynamics and their experimental implications. Journal of Physics A: Mathematical and Theoretical. 2007;40(4):755-764.

4. Gasbarri G, Toros M, Donadi S, et al. Gravity induced Wave Function Collapse. Cornell University Library, USA, 2017. p. 1-19.

5. Bassi A, Lochan K, Satin S, et al. Models of wave-function collapse, underlying theories, and experimental tests". Reviews of Modern Physics. 2013;85(2):471-527.

6. Bassi A, Grossardt A, Ulbricht H. Gravitational Decoherence. Classical and Quantum Gravity. 2017;34:1-78.

7. Pekar SI. Untersuchungen uber die Elektronentheorie der Kristalle. Akademie Verlag, Berlin, Germany, 1954. p. 29-34.

8. Ruffini R, Bonazzola S. Systems of self-gravitating particles in general relativity and the concept of an equation of state. Physical Review Journals Achieves. 1969;187(5).

9. Lieb EH. Existence and uniqueness of the minimizing solution of Choquard's nonlinear equation. Studied in Applied Mathematics. 1977;57(2):93-105.

10. Nawa H, Ozawa T. Nonlinear scattering with nonlocal interaction. Communications in Mathematical Physics. 1922;146(2):259-275.

11. Cazenave T. Semi linear Schrödinger Equations. Oxford University Press, AMS, Courant Institute of Mathematical Sciences, New York, USA. 2003.

12. Kato T. Nonlinear Schrödinger equations. In Schrödinger operators Sonderborg, Lecture Notes in Physics, Springer, Berlin, Germany. 1989;345:218-263.

13. Choquard P, Stubbe J. The one-dimensional Schrödinger-Newton equation. Letters in Mathematical Physics. 2007;81(2):177-184.

14. Jonsson G, Froehlich J, Lars B, et al. Boson Stars and Solitary Waves. Communications in Mathematical Physics. 2007;274(1):1-30.

15. Amor AB, Blanchard P. Local existence and uniqueness for the frictional Newton-Schrödinger equation in three dimensions. Cornell University Library, USA. 2009. p. 1-19. 
16. Bahrami M, Grossardt A, Donadi S, et al. The Schrödinger-Newton equation and its foundations. New Journal of Physics. 2014;16: 115007115025.

17. Giulini D, Grossardt A. The Schrödinger-Newton equation as non relativistic limit of gravitating Klein-Gordon and Dirac fields. Classical and Quantutam Gravity. 2012;29(21):215010.

18. Xu X-J. Pseudo differential operators and their applications. Lecture notes for 8th summer school on nonlinear PDE analysis Pseudo differential Operators and Their Applications. Wuhan University, China. 2010.

19. Farkas EW. Function spaces of generalized smoothness and pseudodifferential operators associated to a continuous negative definite function. Faculty of Mathematics, Computer Science and Statistics, Ludwig-Maximilian University of Munich, Germany. 2002. p. 1-151.

20. El-Nabulsi RA. Calculus of variations with hyper differential operators from Tabasaki-Takebe-Toda lattice arguments. Revista de la Real Academia de Ciencias Exactas, Fisicas y Naturales. Serie A Matematicas. 2013;107(2):419-436.

21. Anastopoulos $\mathrm{C}, \mathrm{Hu} \mathrm{BL}$. Problems with the Newton-Schrödinger equations. New Journal of Physics. 2014;16:1-20.

22. Moroz I, Penrose R, Tod KP. Spherically-symmetric solutions of the Schrödinger-Newton equations. Classical and Quantum Gravity. 1998;15(9)

23. Harrison R, Moroz I, Tod KP. A numerical study of the SchrödingerNewton equations. Nonlinearity. 2003;16(1).

24. Bernstein DH, Giladi E, Jones KRW. Eigenstates of the Gravitational Schrödinger Equation. Modern Physics Letters A. 1998;13(29):23272336 .

25. Illner R, Zwiefel PF, Lange H. Global existence, uniqueness and asymptotic behavior of solutions of the Wigner-Poisson and SchrödingerPoisson systems. Mathematical Methods in the Applied Sciences. 1994;17(5):349-376.

26. Lange H, Toomire B, weifel PFZ. An overview of Schrödinger-Poisson Problems. Reports on Mathematical Physics. 1995;36(2-3): 331-345.

27. Tod KP. The ground state energy of the Schrödinger-Newton equation. Physics Letters A. 2001;280(4):173-176.

28. Melko R, Mann R. Studies of the Schrödinger-Newton Equations in Dimensions. Cornell University Library, USA, 2002. p. 1-18.

29. Weinberg S. Quantum Mechanics without State vectors. Physical Review A. 2014;90(4).

30. Lindblad G. On the generators of quantum dynamical semi groups. Communications in Mathematical Physics. 1976;48(2):119-130.

31. Barandes JA, Kagan D. The Minimal Modal Interpretation of Quantum Theory. Cornell University Library, USA. 2014.

32. Makela J. Schrödinger Equation of the Schwarzschild Black Hole. Cornell University Library, USA. 1996.
33. Spallucci E, Smailagic AA. Regular black holes from semi-classical down to Planckian size. International Journal of Modern Physics D. 2017;26(7):1730013-1730040.

34. Spallucci E, Smailagic A. Gaussian black holes in Rastall Gravity. Cornell University Library, USA. 2017.

35. Harrison R, Moroz I, Tod K. A numerical study of the Schrödinger-Newton equation 1: Perturbing the spherically symmetric stationary states. Cornell University Library, USA, 2002. p. 1-20.

36. Manfredi G, Hervieux PA, Haas F. Variational approach to the timedependent Schrödinger-Newton equations. Classical and Quantum Gravity. 2013;30(7).

37. Salzman PJ, Carlip S. A possible experimental test of quantized gravity. Cornell University Library, USA. 2006.

38. Guzman FS, Urena-Lopez LA. Evolution of the Schrödinger-Newton system for a self-gravitating scalar field. Physical Review D. 2004;69(12).

39. Meter JRV. Schrödinger-Newton "collapse" of the wave function. Classical and Quantum Gravity. 2011;28(1).

40. Modesto L. Super-renormalizable quantum gravity. Physical Review D. $2012 ; 86(4)$

41. Buoninfante L. Ghost and singularity free theories of gravity. Cornell University Library, USA. 2016.

42. Biswas T, Gerwick E, Koivisto T, et al. Towards Singularity- and GhostFree Theories of Gravity. Physical Review Letter. 2012;108(3).

43. Buoninfante L, Lambiase G, Mazumdar A. Quantum spreading of a selfgravitating wave-packet in singularity free gravity. The European Physical Journal C. 2018;78(1).

44. Buoninfante L, Lambiase G, Mazumdar A. Quantum solitonic wavepacket of a meso-scopic system in singularity free gravity. Cornell University Library, USA. 2017.

45. Myung YS, Park YJ. The origin of regular Newtonian potential in infinite derivative gravity. Cornell University Library, USA. 2017.

46. Santamato E. Geometric derivation of the Schrödinger equation from classical mechanics in curved Weyl spaces. Physical Review D. 1984;29(2).

47. Santamato E. Statistical interpretation of the Klein-Gordon equation in terms of the space-time Weyl curvature. Journal of Mathematical Physics. $1984 ; 25(8)$

48. C Castro. Nonlinear Quantum Mechanics as the Weyl Geometry of a Classical Statistical Ensemble. Foundation Physics Letters. 1991;4(1):8199.

49. Castro C. On Weyl Geometry, Random Processes and Geometric Quantum Mechanics. Foundations of Physics. 1992;22(4):569-615.

50. Tavernelli I. On the Geometrization of Quantum Mechanics. Annals of Physics. 2016;371:239-253.

51. Castro C. An Elegant solution to the Cosmological Constant Problem from the Bohm-Poisson Equation. 2017. p. 1-6. 